\title{
Factors Associated with Fibrosis during Colorectal Endoscopic Submucosal Dissection: Does Pretreatment Biopsy Potentially Elicit Submucosal Fibrosis and Affect Endoscopic Submucosal Dissection Outcomes?
}

\author{
Masatake Kuroha ${ }^{a}$ Hisashi Shiga ${ }^{a}$ Yoshitake Kanazawa ${ }^{a}$ Hiroshi Nagai ${ }^{a}$ \\ Tomoyuki Handa ${ }^{a}$ Ryo Ichikawa ${ }^{a}$ Motoyuki Onodera ${ }^{a}$ Takeo Naito ${ }^{a}$ \\ Rintaro Moroi $^{a}$ Tomoya Kimura $^{a}$ Katsuya Endob Yoichi Kakuta ${ }^{\mathrm{a}}$ \\ Yoshitaka Kinouchi $^{c}$ Tooru Shimosegawa ${ }^{a}$ Atsushi Masamune ${ }^{a}$ \\ ${ }^{a}$ Division of Gastroenterology, Tohoku University Graduate School of Medicine, Sendai, Japan; bepartment of \\ Gastroenterology, Tohoku Medical and Pharmaceutical University, Sendai, Japan; ${ }^{C}$ Health Administration Center, \\ Center for the Advancement of Higher Education, Tohoku University, Sendai, Japan
}

\section{Keywords}

Adenoma - Adenocarcinoma - Colorectal cancer .

Endoscopic mucosal resection · Laterally spreading tumor

\begin{abstract}
Background: Submucosal fibrosis observed during colorectal endoscopic submucosal dissection (ESD) is an important factor related to incomplete resection. Biopsy is generally accepted as having the potential to elicit submucosal fibrosis, but few reports have presented definitive proof. This study investigated the relation between submucosal fibrosis and colorectal ESD outcomes and assessed factors related to fibrosis, including pretreatment biopsy. Methods: After reviewing 369 records of colorectal ESD performed between January 2011 and December 2016, we assessed the relation between fibrosis and ESD outcomes. Multiple logistic regression analysis revealed fibrosis risk factors. Results: Severe fibrosis was related significantly to ESD outcomes such as the mean procedure time $(p<0.001)$, en bloc resection rate $(p<$ $0.001)$, and $\mathrm{R} 0$ resection rate $(p=0.011)$. Multivariate analyses
\end{abstract}

karger@karger.com www.karger.com/dig

Karger $\frac{1}{6}$

BOPEN ACCESS
(C) 2020 The Author(s)

Published by S. Karger AG, Basel

This article is licensed under the Creative Commons AttributionNonCommercial-NoDerivatives 4.0 International License (CC BYNC-ND) (http://www.karger.com/Services/OpenAccessLicense) Usage and distribution for commercial purposes as well as any distribution of modified material requires written permission. indicated residual lesions (ORs 175.4, $p<0.001$ ), pretreatment biopsy (ORs 8.30, $p=0.002$ ), nongranular-type laterally spreading tumors (LST-NG; ORs 5.86, $p=0.025$ ), and invasive carcinoma (ORs 5.83, $p=0.03$ ) as independent risk factors of severe fibrosis. In each macroscopic type, LST-NG was more strongly related to fibrosis induced by pretreatment than granular-type laterally spreading tumors with adjust ORs of 50.8 and 4.69. Conclusions: Pretreatment biopsy causes submucosal fibrosis resulting in prolonged procedure times and incomplete resection. These findings suggest important benefits of avoiding biopsy before ESD.

(C) 2020 The Author(s)

Published by S. Karger AG, Basel

\section{Introduction}

Colorectal endoscopic submucosal dissection (ESD) is gradually gaining widespread acceptance worldwide [1$3]$. This technique permits en bloc removal of tumors of $>2 \mathrm{~cm}$ in diameter, leading to precise histological assessment and a lower recurrence rate than that obtained using 

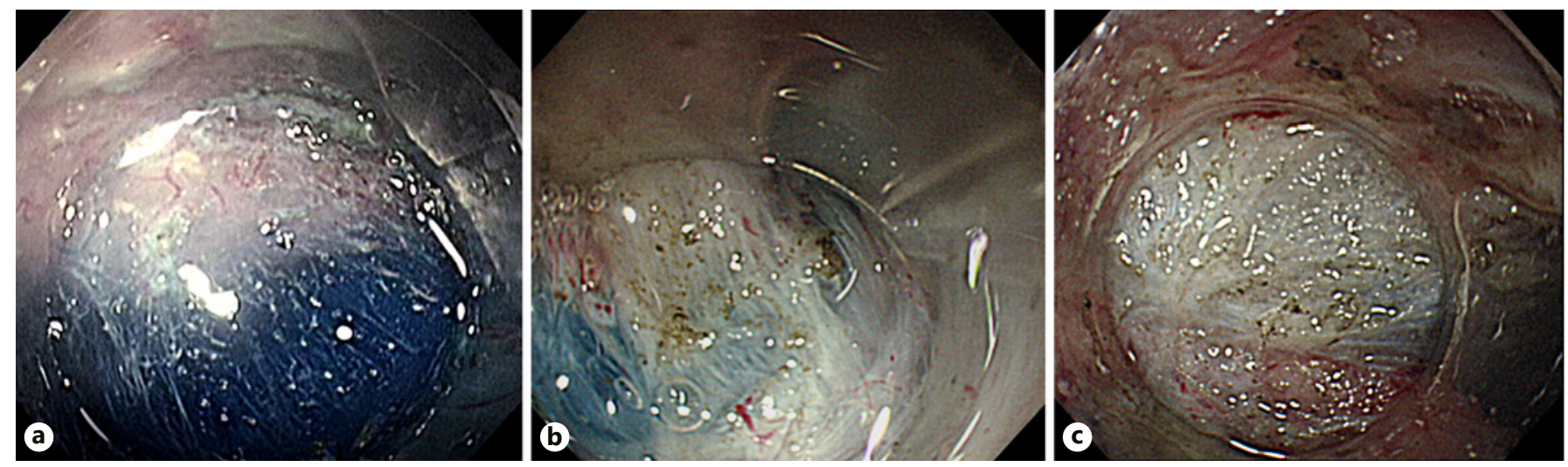

Fig. 1. Degree of fibrosis of the submucosal layers during colorectal ESD. No fibrosis: which manifested as a blue transparent layer. Mild fibrosis: which appears as a white web-like structure in the blue submucosal layers. Severe fibrosis: which appears as a white muscular structure without blue transparent layer in the submucosal layers.

endoscopic mucosal resection (EMR) [4]. Nevertheless, because of anatomical features of the organs such as a thin colonic wall and the presence of peristalsis leading to complications such as perforation during procedures and longer procedure times, colorectal ESD presents a higher degree of technical difficulty than gastric ESD $[5,6]$.

Reportedly, predictors of incomplete resection include the experience of endoscopists [7], paradoxical movement of the colonoscope [8], tumor size [6], tumor location [9], and submucosal fibrosis $[6,8,10,11]$. Among them, submucosal fibrosis is an important factor related to incomplete resection. Japanese Society of Gastroenterological Endoscope (JSGE) guidelines state the indications of EMR and ESD for colorectal lesions [12]. The guidelines state that mucosal tumors with submucosal fibrosis caused by pretreatment biopsy indicate ESD because EMR is difficult as a result of nonlifting sign. The guideline also states that biopsy is not recommended at the lesion because biopsy potentially elicits submucosal fibrosis leading to a positive nonlifting sign, which makes subsequent endoscopic treatment difficult. Moreover, for large lesions such as granular laterally spreading tumor (LST-G), which is "carcinoma in adenoma" in many cases, biopsy might not support accurate diagnosis in many cases. These findings are widely accepted by gastroenterologists in Japan. Nevertheless, few studies have specifically examined the association between pretreatment biopsy and submucosal fibrosis observed during colorectal ESD. In the MINDS Grade of Recommendations [13], the evidence level related to pretreatment biopsies is rated as $\mathrm{V}$ (Descriptive study). The grade of recommendations is rated as $\mathrm{C} 1$. No scientific evidence exists, but therapy is recommended. In addition, biopsy capabilities for distin- guishing adenocarcinoma from adenoma have been little reported.

This study evaluated the relation between submucosal fibrosis observed during colorectal ESD and colonic ESD outcomes. We evaluated factors associated with submucosal fibrosis and investigated whether pretreatment biopsy affected the fibrosis of the submucosal layer during ESD. We also examined whether adenocarcinoma is distinguishable from adenoma using pretreatment biopsy.

\section{Methods}

\section{Patients}

For this study, we reviewed ESD records of 369 colorectal lesions in 355 patients treated at Tohoku University Hospital between January 2011 and December 2016. This retrospective study was approved by the Ethics Committee of Tohoku University Hospital (No. 20161-003). All the patients provided written informed consent.

\section{Indication Criteria for Colorectal ESD}

We included colorectal lesions indicated by preoperative endoscopic findings as $>20 \mathrm{~mm}$ in diameter. Indication criteria for colorectal ESD were based on JSGE guidelines as follows: (1) lesions for which endoscopic en bloc resection is required, that is, lesions for which en bloc resection with EMR is difficult such as nongranular-type laterally spreading tumor (LST-NG), lesions with a VI-type pit pattern, carcinoma with superficial invasion, large depressed-type tumors, and large protruded-type lesions suspected to be carcinoma; (2) mucosal tumors with submucosal fibrosis; (3) sporadic localized tumors in conditions of chronic inflammation such as ulcerative colitis; and (4) local residual or recurrent early carcinomas after endoscopic resection.

\section{Colorectal ESD Procedures}

Details of colorectal ESD procedures were presented in our earlier report [14]. Following is a brief description of the procedure. 
Pediatric endoscopes were used (PCF-Q260JI and GIF-Q260J; Olympus Optical Co., Tokyo, Japan) with a disposable attachment. Into the submucosal layer, we injected a mixture of solution containing sodium hyaluronate with a small volume of epinephrine and indigocarmine. A tip-type knife (Dual Knife; Olympus Optical Co., Tokyo, Japan) was used for mucosal incision and submucosal dissection. We used an electrosurgical generator (ICC 200; ERBE Elektromedizin GmbH, Tubingen, Germany).

Procedures of colorectal ESD were performed by several endoscopists. All procedures were conducted by trainees under the supervision of experienced endoscopists. In cases of perforation induced by trainees, subsequent procedures were performed by experienced endoscopists.

\section{Endoscopic and Histological Assessments}

Tumor sites were divided into 3 groups: the rectum, left colon (sigmoid colon and descending colon), and right colon (transverse colon, ascending colon, and cecum). Macroscopic tumor types were classified as protruding tumor (Is), granular-type laterally spreading tumor (LST-G; Is + IIa, IIa), nongranular-type laterally spreading tumor (LST-NG; IIa, IIc), or residual lesions.

After colorectal ESD procedures, the case in which the tumor was resected in a single piece was judged as endoscopic en bloc resection. If the tumor was resected en bloc endoscopically and if the lateral and basal margins were free of tumor cells in histological analysis, the tumor was an intramucosal carcinoma or carcinoma with slight submucosal invasion (invasion depth $<1,000 \mu \mathrm{m}$ ), and there was no lymphatic invasion and vascular involvement, then it was defined as R0 (complete) resection. Other cases were defined as $\mathrm{R} 1$ (incomplete) or $\mathrm{Rx}$ (not evaluable) resection.

Primary ESD outcomes were technical difficulty defined as procedure time and clinical success defined as en bloc resection and R0 resection. Secondary outcomes were complication rates such as delayed bleeding and perforation.

Based on findings obtained using injection of indigo carmine solution into the submucosal layer, the degree of submucosal fibrosis was classified as follows (Fig. 1): no fibrosis, mild fibrosis, and severe fibrosis [15]. Three experienced endoscopists independently reviewed the degree of submucosal fibrosis during ESD without information about the patients' background including the presence or absence of pretreatment biopsy, tumor histopathological types, or previous history of EMR. Sporadic localized lesions with inflammatory bowel disease such as ulcerative colitis are known to have a high rate of fibrosis in the submucosal layer [16]. Consequently, we exclude these conditions from this study.

Whether biopsy was performed and the number and location of biopsy in the lesion were decided before visiting our hospital by doctors who had no experience of colorectal ESD. For that reason, no standard criteria for pretreatment biopsy were found in our study. In many cases, biopsy was performed with 1 or 2 pieces from the central part of the lesion. We defined cases with biopsy within 3 months of colorectal ESD as pretreatment biopsy lesion.

Histopathological diagnoses were based on the Japanese classification of colorectal cancer [17]. Intramucosal carcinoma was defined as noninvasive carcinoma. Carcinoma that invades into the submucosal layer was defined as invasive carcinoma. Depth of tumor invasion was the distance between the deeper edge of the muscularis mucosae and the deepest invasion. Biopsy specimens were classified as follows: normal tissue lesion, material for which diagnosis of neoplastic or nonneoplastic lesion is difficult, adenoma, neoplastic lesion
Table 1. Clinical characteristics of patients

\begin{tabular}{lc}
\hline & 355 patients, \\
& 369 lesions \\
\hline Age, years, mean (SD) & $68.4(10.6)$ \\
Sex, male/female, $n$ & $218 / 137$ \\
Mean tumor diameter, mm (SD) & $44.1(18.1)$ \\
$\quad<40$ mm & 203 \\
$\geq 40$ mm & 166 \\
Location, $n(\%)$ & \\
Rectum & $99(26.8)$ \\
Left colon & $59(16.0)$ \\
Right colon & $211(57.2)$ \\
Macroscopic type, $n(\%)$ & \\
LST-G & $181(49.1)$ \\
LST-NG & $102(27.6)$ \\
Protruding & $63(17.1)$ \\
Depressed & $9(2.4)$ \\
Residual & $14(3.8)$ \\
Pretreatment biopsy,$n(\%)$ & $63(17.1)$ \\
Mean number of biopsies per lesion & 1.8 \\
Fibrosis, $n(\%)$ & \\
No fibrosis & $276(74.8)$ \\
Mild fibrosis & $72(19.5)$ \\
Severe fibrosis & $21(5.7)$ \\
Histological type, $n(\%)$ & $188(51.0)$ \\
Adenoma & $124(33.6)$ \\
Noninvasive carcinoma & $57(15.4)$ \\
Invasive carcinoma & \\
\hline
\end{tabular}

LST-G, laterally spreading tumor granular type; LST-NG, laterally spreading tumor nongranular type; SD, standard deviation. ${ }^{a}$ Lesion biopsied within 3 months of colorectal ESD.

suspected of being carcinoma, and carcinoma. The pretreatment diagnosis from biopsy was regarded as carcinoma in cases with neoplastic lesion suspected of being carcinoma or carcinoma.

\section{Statistical Analysis}

Quantitative data are presented as mean with standard deviation (SD). All statistical analysis was done using software (JMP ver. 13; SAS Institute Inc., Cary, NC, USA). Differences among groups were evaluated using the $\chi^{2}$ test or Fisher's exact probability test, as appropriate. Among the clinical characteristics, factors influencing the main outcome were identified using a multiple logistic regression method. Odds ratios (ORs) and 95\% confidence intervals (CIs) were calculated to assess the strength of the influence of each variable. Significance was inferred for $p<0.05$.

\section{Results}

\section{Patient and Lesion Characteristics}

Three hundred seventy-two colorectal tumors were enrolled in this study. Three patients were excluded because 

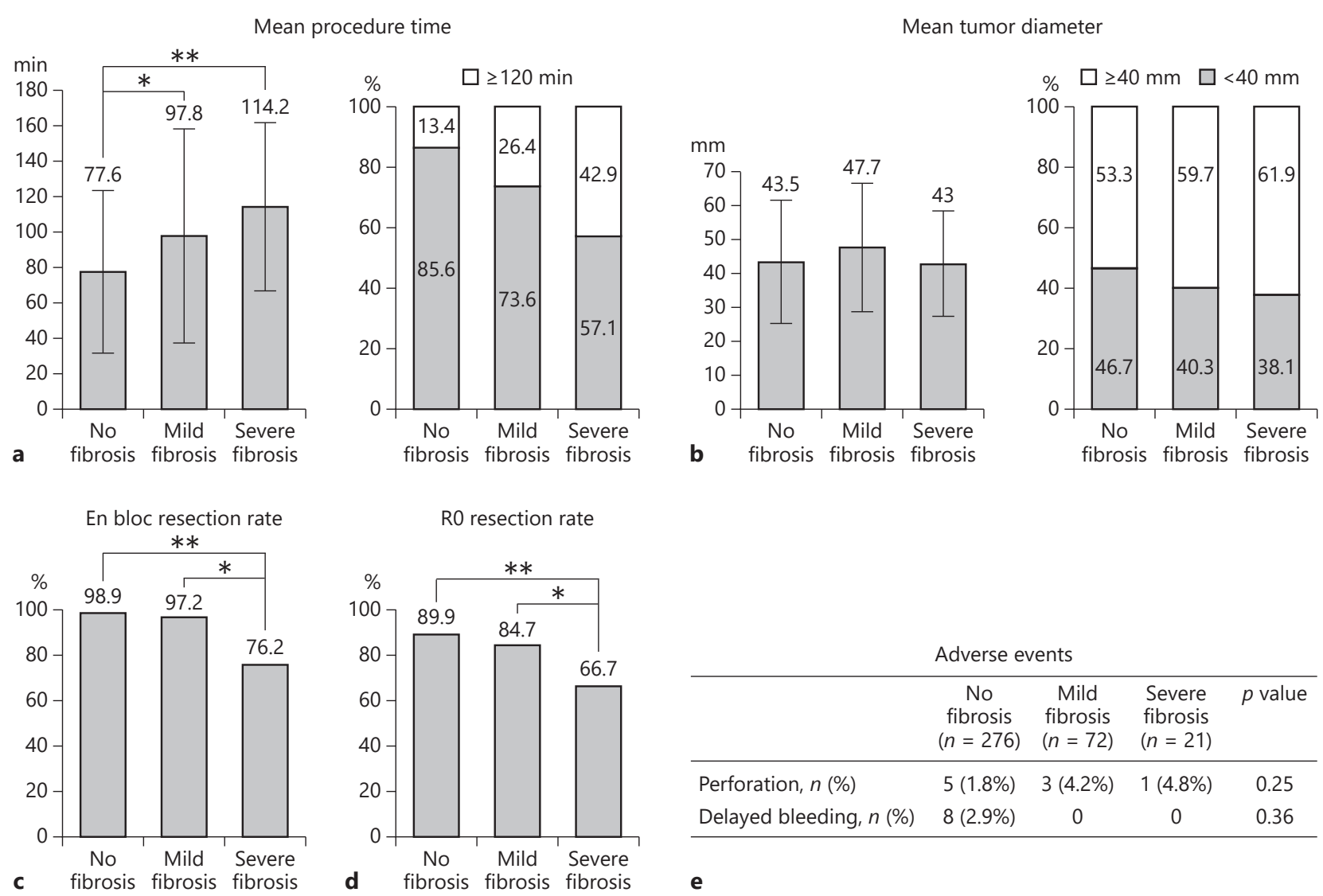

\begin{tabular}{lcccc} 
& Adverse events & & \\
\hline & $\begin{array}{c}\text { No } \\
\text { fibrosis } \\
(n=276)\end{array}$ & $\begin{array}{c}\text { Mild } \\
\text { fibrosis } \\
(n=72)\end{array}$ & $\begin{array}{c}\text { Severe } \\
\text { fibrosis } \\
(n=21)\end{array}$ & $p$ value \\
\hline Perforation, $n(\%)$ & $5(1.8 \%)$ & $3(4.2 \%)$ & $1(4.8 \%)$ & 0.25 \\
Delayed bleeding, $n(\%)$ & $8(2.9 \%)$ & 0 & 0 & 0.36 \\
\hline
\end{tabular}

Fig. 2. ESD outcomes. Left side: mean procedure times were 77.6 min in the nonfibrosis group, $97.8 \mathrm{~min}$ in the mild fibrosis group, and $114.2 \mathrm{~min}$ in the severe fibrosis group. Mean procedure times were significantly longer in the severe fibrosis group and mild fibrosis group than in the nonfibrosis group. ${ }^{*} p=0.01,{ }^{* *} p<0.001$. Right side: proportion of ESD procedure times over $120 \mathrm{~min}$. Left side: mean tumor diameter in each fibrosis group. No significant differences were found among the 3 groups. Right side: proportion of tumor diameter over $40 \mathrm{~mm}$. En bloc resection rates were $98.9 \%$ in the nonfibrosis group, $97.2 \%$ in the mild fibrosis group, and $76.2 \%$ in the severe fibrosis group. The en bloc resection rate was

of sporadic localized lesions with ulcerative colitis. Clinicopathological characteristics of the enrolled 369 colorectal tumors are presented in Table 1 . Three hundred sixtynine lesions from 218 men and 137 women were reviewed. The mean age of the patients was $68.4( \pm 10.6)$ years. The mean colorectal tumor diameter was $44.1 \mathrm{~mm}$. Locations of lesions were divided, respectively, into those of the rectum, the left colon, and the right colon in 99 (26.8\%), 59 (16.0\%), and 211 (57.2\%) lesions. Macroscopic-type lesions were divided, respectively, into 181 (49.1\%) LST-G, significantly lower in the severe fibrosis group than in the mild fibrosis group or nonfibrosis group. ${ }^{*} p=0.004,{ }^{* *} p<0.001$. R0 resection rates were $89.9 \%$ in the nonfibrosis group, $84.7 \%$ in the mild fibrosis group, and $66.7 \%$ in the severe fibrosis group. The R0 resection rate was significantly lower in the severe fibrosis group than in the mild fibrosis group and nonfibrosis group. ${ }^{*} p=0.047,{ }^{* *} p<$ 0.003 . If the tumor was resected en bloc endoscopically and if the lateral and basal margins were free of tumor cells, it was defined as R0 (complete) resection. Other cases were defined as R1 (incomplete) or Rx (not evaluable) resection. Adverse events in respective fibrosis groups. No significant difference was found among groups.
102 (27.6\%) LST-NG, 63 (17.1\%) protruding, 9 (2.4\%) depressed, and $14(3.8 \%)$ residual lesions. Pretreatment biopsy was performed in 63 lesions (17.1\%). In these cases, the mean number of biopsies taken from a lesion was 1.8. Additionally, 276 lesions (74.8\%) were in the nonfibrosis group, 72 lesions (19.5\%) were in the mild fibrosis group, and 21 lesions (5.7\%) were in the severe fibrosis group. The histopathological diagnosis was adenoma in 188 lesions (51.0\%), noninvasive carcinoma in 124 lesions (33.6\%), and invasive carcinoma in 57 lesions (15.4\%). 
Table 2. Risk factors associated with mild fibrosis during ESD procedure

\begin{tabular}{|c|c|c|c|c|c|c|}
\hline & \multirow[t]{2}{*}{ Mild fibrosis } & \multirow{2}{*}{$\begin{array}{l}\text { Univariate* }^{*} \\
p \text { value }\end{array}$} & \multicolumn{4}{|c|}{ Multivariate** } \\
\hline & & & ORs & $p$ value & $95 \% \mathrm{C}$ & \\
\hline \multicolumn{7}{|l|}{ Location, $n(\%)$} \\
\hline Rectum & $12 / 91(13.2)$ & 0.14 & Reference & & & \\
\hline Left colon & $12 / 55(21.8)$ & & 2.62 & 0.08 & 0.89 & 7.65 \\
\hline Right colon & $48 / 202(23.8)$ & & 4.03 & 0.002 & 1.70 & 9.57 \\
\hline \multicolumn{7}{|l|}{ Macroscopic type, $n(\%)$} \\
\hline LST-G & 26/177 (14.7) & 0.01 & Reference & & & \\
\hline LST-NG & $25 / 97(27.9)$ & & 2.18 & 0.03 & 1.08 & 4.39 \\
\hline Protruding & $16 / 61(26.2)$ & & 2.49 & 0.025 & 1.12 & 5.53 \\
\hline Depressed & $1 / 7(14.3)$ & & 0.47 & 0.52 & 0.05 & 4.65 \\
\hline Residual & $4 / 6(66.7)$ & & 14.4 & 0.008 & 2.04 & 101.3 \\
\hline \multicolumn{7}{|l|}{ Histological type, $n(\%)$} \\
\hline Adenoma & $30 / 182(16.5)$ & 0.011 & Reference & & & \\
\hline Noninvasive carcinoma & $24 / 116(20.7)$ & & 1.69 & 0.14 & 0.84 & 3.38 \\
\hline Invasive carcinoma & $18 / 50(35.6)$ & & 3.51 & 0.03 & 1.55 & 7.93 \\
\hline \multicolumn{7}{|l|}{ Tumor size, $n(\%)$} \\
\hline$<40 \mathrm{~mm}$ & $29 / 158(18.4)$ & 0.33 & & & & \\
\hline$\geq 40 \mathrm{~mm}$ & $43 / 190(22.6)$ & & & & & \\
\hline \multicolumn{7}{|l|}{ Pretreatment biopsy, $n(\%)$} \\
\hline Absent & $46 / 294(15.7)$ & $<0.001$ & Reference & & & \\
\hline Present & $26 / 54(48.2)$ & & 7.21 & $<0.001$ & 3.51 & 14.8 \\
\hline
\end{tabular}

CI, confidence interval; LST-G, laterally spreading tumor granular type; LST-NG, laterally spreading tumor nongranular type. ${ }^{*} \chi^{2}$ test or Fisher's exact probability test was used. ${ }^{* *}$ Variables with a $p$ value of $<0.2$ in univariate analysis were included in a multiple logistic regression method.

\section{Outcomes of ESD}

The ESD outcomes are presented in Figure 2. The mean procedure times were $77.6 \mathrm{~min}$ in the nonfibrosis group, $97.8 \mathrm{~min}$ in the mild fibrosis group, and $114.2 \mathrm{~min}$ in the severe fibrosis group (Fig. 2a). The mean procedure time was significantly longer in either the severe fibrosis group or the mild fibrosis group than in the nonfibrosis group. Mean tumor diameter was $43.5 \mathrm{~mm}$ in the nonfibrosis group, $47.7 \mathrm{~mm}$ in the mild fibrosis group, and 43 $\mathrm{mm}$ in the severe fibrosis group (Fig. 2b). No significant difference was found among the 3 groups. Overall en bloc resection and R0 resection rates were 98.9 and $89.9 \%$ in the nonfibrosis group, 97.2 and $84.7 \%$ in the mild fibrosis group, and 76.2 and $66.7 \%$ in the severe fibrosis group (Fig. 2c, d). Rates of en bloc resection and $\mathrm{R} 0$ resection were significantly lower in the severe fibrosis group than in either the mild fibrosis group or the nonfibrosis group. The perforation and delayed bleeding rates were 1.8 and $2.9 \%$ in the nonfibrosis group, 4.2 and $0 \%$ in the mild fibrosis group, and 4.8 and $0 \%$ in the severe fibrosis group (Fig. 2e). No significant difference was found among the severe fibrosis, mild fibrosis, and nonfibrosis groups for perforation or delayed bleeding rates.

\section{Risk Factors Associated with Submucosal Fibrosis Observed during Colorectal ESD}

We analyzed possible risk factors of both mild and severe fibrosis. Regarding the mild fibrosis, univariate analyses revealed that macroscopic types, histological types, and pretreatment biopsy were associated significantly with mild fibrosis (Table 2). Multivariate analysis revealed that lesions at the right colon, macroscopic types (LST-NG, protruding, and residual lesions), invasive carcinoma, and pretreatment biopsy were significantly associated, respectively, with mild fibrosis with adjusted ORs of 4.03 (95\% CI: 1.70-9.57), 2.18 (1.08-4.39), 2.49 (1.12-5.53), 14.4 (2.04-101.3), 3.51 (1.55-7.93), and 7.21 (3.51-14.8) (Table 2). Regarding the severe fibrosis, univariate analyses revealed that macroscopic types, histological types, and pretreatment biopsy were associated significantly with severe fibrosis (Table 3 ). Multivariate analysis revealed that macroscopic types (LST-NG and residual lesions), invasive carcinoma, and pretreatment biopsy were associated significantly with severe fibrosis with adjusted ORs of 5.86 (95\% Cl: 1.25-27.4), 175.4 (22.2-1,382.7), 5.83 (11.5-29.6), and 8.30 (2.17-31.7) (Table 3). 
Table 3. Risk factors associated with severe fibrosis during ESD procedure

\begin{tabular}{|c|c|c|c|c|c|c|}
\hline & \multirow[t]{2}{*}{ Severe fibrosis } & \multirow{2}{*}{$\begin{array}{l}\text { Univariate* } \\
p \text { value }\end{array}$} & \multicolumn{4}{|c|}{ Multivariate ${ }^{* *}$} \\
\hline & & & ORs & $p$ value & $95 \% \mathrm{C}$ & \\
\hline \multicolumn{7}{|l|}{ Location, $n(\%)$} \\
\hline Rectum & $8 / 87(9.2)$ & 0.51 & & & & \\
\hline Left colon & $4 / 47(8.5)$ & & & & & \\
\hline Right colon & $9 / 163(5.5)$ & & & & & \\
\hline \multicolumn{7}{|l|}{ Macroscopic type, $n(\%)$} \\
\hline LST-G & $4 / 155(2.6)$ & $<0.001$ & Reference & & & \\
\hline LST-NG & $4 / 77(6.5)$ & & 5.86 & 0.025 & 1.25 & 27.4 \\
\hline Protruding & $2 / 47(4.3)$ & & 1.35 & 0.74 & 0.22 & 8.47 \\
\hline Depressed & $2 / 8(25.0)$ & & 5.86 & 0.22 & 0.41 & 45.7 \\
\hline Residual & $8 / 10(80.0)$ & & 175.4 & $<0.001$ & 22.2 & $1,382.7$ \\
\hline \multicolumn{7}{|l|}{ Histological type, $n(\%)$} \\
\hline Adenoma & $6 / 158(3.8)$ & 0.007 & Reference & & & \\
\hline Noninvasive carcinoma & $8 / 100(8.0)$ & & 2.55 & 0.18 & 0.64 & 10.3 \\
\hline Invasive carcinoma & $7 / 39(18.0)$ & & 5.83 & 0.03 & 11.5 & 29.6 \\
\hline \multicolumn{7}{|l|}{ Tumor size, $n(\%)$} \\
\hline$<40 \mathrm{~mm}$ & $8 / 137(5.8)$ & 0.44 & & & & \\
\hline$\geq 40 \mathrm{~mm}$ & $13 / 160(8.1)$ & & & & & \\
\hline \multicolumn{7}{|l|}{ Pretreatment biopsy, $n(\%)$} \\
\hline Absent & $12 / 260(4.6)$ & $<0.001$ & Reference & & & \\
\hline Present & 9/37 (24.3) & & 8.30 & 0.002 & 2.17 & 31.7 \\
\hline
\end{tabular}

Table 4. Association between pretreatment biopsy and fibrosis in respective macroscopic types

\begin{tabular}{|c|c|c|c|c|c|c|c|c|c|}
\hline Pretreatment biopsy & LST-NG & $p$ value & ORs & LST-G & $p$ value & ORs & Protruding & $p$ value & ORs \\
\hline \multicolumn{10}{|c|}{ Fibrosis (mild and severe) } \\
\hline Absent & $18 / 88$ & & & $21 / 144$ & & & $15 / 51$ & & \\
\hline Present & $14 / 15$ & $<0.001$ & 50.8 & $16 / 36$ & $<0.001$ & 4.69 & $4 / 9$ & 0.37 & 1.92 \\
\hline
\end{tabular}

LST-G, laterally spreading tumor granular type; LST-NG, laterally spreading tumor nongranular type.

To reveal the relation between fibrosis and pretreatment biopsy in each macroscopic type, subgroup analyses were conducted, respectively, for 3 major macroscopic types (LST-NG, LST-G, and protruding type) (Table 4). Depressed-type and residual lesions were excluded because of their few numbers. Because the severe fibrosis group was small and we specifically examined not the degree of fibrosis but the presence or absence of fibrosis, cases were divided into 2 groups as the no fibrosis and fibrosis groups (including mild fibrosis and severe fibrosis) in this subanalysis. Results of pretreatment biopsy revealed that LST-NG and LST-G were associated significantly with increased frequencies of fibrosis (mild and severe). The ORs of fibrosis induced by pretreatment biopsy were estimated, respectively, as 50.8 and 4.69 for LST-NG and LST-G.

\section{Can Adenocarcinoma Be Distinguished from}

Adenoma by Pretreatment Biopsy?

Next, we examined whether adenocarcinoma is distinguishable from adenoma by histopathological assessment of biopsy specimens (Table 5). We compared the pretreatment diagnoses based on biopsy specimens and the histologic diagnoses of ESD specimens. Among the 17 lesions with biopsy sampling diagnoses of carcinoma, 15 (88.2\%) were diagnosed as carcinoma. Among the 45 le- 
Table 5. Comparison of diagnosis based on pretreatment biopsy specimens and histologic diagnosis of resected specimens

Histological diagnosis of resected specimens carcinoma adenoma

Pretreatment diagnosis based on biopsy specimens Carcinoma $\quad 15 \quad 2$ Adenoma $\quad 26 \quad 19$

sions with biopsy diagnoses of adenoma, 26 (57.8\%) were diagnosed as carcinomas. The biopsy sampling predicted final histologic diagnoses of carcinoma with a sensitivity of $36.6 \%$, specificity of $90.5 \%$, accuracy of $54.8 \%$, positive predictive value of $88.2 \%$, and negative predictive value of $42.2 \%$.

\section{Discussion}

Results of our study demonstrated that the presence of severe fibrosis in submucosal is associated with a low en bloc resection rate and $\mathrm{R} 0$ resection rate. Moreover, the mean procedure time was significantly longer in both mild and severe fibrosis groups. Similarly to our result, earlier reports have described that the presence of submucosal fibrosis is associated with a low en bloc resection rate $(63.1-77.4 \%)$ and extension of the mean resection time $[6,11,18,19]$.

In the present study, the most important risk factors of both severe fibrosis and mild fibrosis were residual lesions (severe fibrosis: ORs 175.4, mild fibrosis: ORs 14.4). The second important risk factor was pretreatment biopsy (severe fibrosis: ORs 8.30, mild fibrosis: ORs 7.21). Few studies have examined the association of submucosal fibrosis and pretreatment biopsy [2022]. Kim et al. [18] reported a lack of association between pretreatment biopsy and submucosal fibrosis in 158 cases (91 cases of biopsy). Takeuchi et al. [23] reported pretreatment biopsy as not a risk factor for nonlifting sign at submucosal injection in 816 cases (446 cases of biopsy). Recently, Fukunaga et al. [24] reported pretreatment biopsy in a flat lesion such as LSTs may cause submucosal fibrosis. Results of the present study revealed that the effects of biopsy vary depending on the macroscopic type. Specifically, LST-NG was more strongly related to fibrosis (ORs 50.8). Moreover, LSTG (ORs 4.69) had less influence from biopsy than LSTNG had. Protruding type showed no biopsy effect. Be- cause LST-NG is a flat lesion, one might infer that biopsy reaches a deeper layer of the submucosa than other macroscopic types, resulting in regenerative changes and subsequent submucosal fibrosis.

This study demonstrated LST-NG and protruding types as independent risk factors for mild fibrosis with adjusted ORs of $2.18(p=0.03)$ and $2.49(p=0.025)$. Moreover, LST-NG was associated significantly with severe fibrosis with adjusted ORs of $5.86(p=0.025)$. Although the depressed type is apparently a risk factor of fibrosis, we cannot assess the associations with fibrosis because few cases were examined in our study. Inada et al. [25] reported that macroscopic types such as LST-NGpseudo-depressed and protruding type, which have $>4$ $\mathrm{cm}$ diameter, are risk factors for submucosal fibrosis. Takeuchi et al. [23] also reported the frequency of a nonlifting sign for LST-NG, protruding type, and LST-nodular mixed type (GM), respectively, as 38,32 , and $16 \%$. A report has described a study demonstrating histologically verified submucosal fibrosis. Reportedly, microvessel density is higher in LST-NG than in LST-G; not only microvessel density but also the degree of fibrosis is higher in LST-NG than in LST-G, particularly in adenoma [26, 27]. These results are regarded as the same as those attained from our study.

This study examined whether biopsy is useful for distinguishing adenocarcinoma from adenoma. The biopsy sampling predicted final histologic diagnoses of carcinoma with a sensitivity of $36.6 \%$, specificity of $90.5 \%$, and accuracy of $54.8 \%$. Several studies demonstrated narrow band imaging (NBI) and/or chromoendoscopy can distinguish neoplastic from nonneoplastic polyps. Kato et al. [28] have shown the accuracy of chromoendoscopy for diagnosing as carcinoma is 99.1\%. Hayashi et al. [29] showed that NBI patterns can be used to predict submucosal invasion in colonic lesions, confirming magnifying chromoendoscopy and/ or NBI as a highly effective method for predicting carcinoma in colorectal neoplasms.

Our study has several limitations. First, it is a retrospective study that enrolled few cases from a single center. Second, we evaluated the degree of fibrosis based on endoscopic findings but not on the histopathology. Lee et al. [19] reported the association between ESD outcomes and fibrosis using histopathological indicators. However, many reports have described diagnoses of fibrosis using endoscopic findings. Moreover, the frequency of fibrosis differs among reports (5.4-36.5\% of cases in the studies). Third, because biopsy was performed before visiting our hospital, details of the biopsy site in the lesion were un- 
clear in many cases. Therefore, it was impractical to verify that the biopsy site and the fibrosis site were truly matched in the lesion.

In conclusion, results of this study demonstrate that pretreatment biopsy causes submucosal fibrosis, thereby resulting in longer procedure times, lower en bloc resection rates, and lower R0 resection rates. Those findings were particularly noticeable in flat-type tumors such as LST-NG. The accuracy of pretreatment specimens for differentiating adenocarcinoma from adenoma is low. The evidence presented above suggests important benefits of avoiding biopsy before ESD.

\section{Statement of Ethics}

This study was approved by the Ethics Committee of Tohoku University Hospital (number: 2016-1-003). All the patients provided written informed consent.

\section{Conflict of Interest Statement}

The authors have no conflicts of interest, financial or otherwise, related to this article or the study it describes.

\section{Funding Sources}

The author received no specific funding for this work.

\section{Author Contributions}

Masatake Kuroha designed the study and wrote the initial draft of the manuscript. Hisashi Siga and Yoshitake Kanazawa contributed to analysis and interpretation of data and assisted in the preparation of the manuscript. All other authors have contributed to data collection and interpretation and critically reviewed the manuscript. All authors approved the final version of the manuscript and agree to be accountable for all aspects of the work in ensuring that questions related to the accuracy or integrity of any part of the work are appropriately investigated and resolved.

\section{References}

1 Tanaka S, Terasaki M, Kanao H, Oka S, Chayama K. Current status and future perspectives of endoscopic submucosal dissection for colorectal tumors. Dig Endosc. 2012; 24(Suppl 1):73-9.

2 Saito Y, Uraoka T, Yamaguchi Y, Hotta K, Sakamoto N, Ikematsu H, et al. A prospective, multicenter study of 1111 colorectal endoscopic submucosal dissections (with video). Gastrointest Endosc. 2010;72(6):1217-25.

3 Saito Y, Bhatt A, Matsuda T. Colorectal endoscopic submucosal dissection and its journey to the West. Gastrointest Endosc. 2017;86(1): 90-2.

4 Saito Y, Fukuzawa M, Matsuda T, Fukunaga S, Sakamoto T, Uraoka T, et al. Clinical outcome of endoscopic submucosal dissection versus endoscopic mucosal resection of large colorectal tumors as determined by curative resection. Surg Endosc. 2010;24(2):343-52.

5 Niimi K, Fujishiro M, Kodashima S, Goto O, Ono S, Hirano K, et al. Long-term outcomes of endoscopic submucosal dissection for colorectal epithelial neoplasms. Endoscopy. 2010;42(9):723-9.

6 Isomoto H, Nishiyama H, Yamaguchi N, Fukuda E, Ishii $\mathrm{H}$, Ikeda $\mathrm{K}$, et al. Clinicopathological factors associated with clinical outcomes of endoscopic submucosal dissection for colorectal epithelial neoplasms. Endoscopy. 2009;41(8):679-83.

7 Shiga H, Ohba R, Matsuhashi T, Jin M, Kuroha M, Endo K, et al. Feasibility of colorectal endoscopic submucosal dissection (ESD) carried out by endoscopists with no or little experience in gastric ESD. Dig Endosc. 2017; 29(Suppl 2):58-65.

Factors Associated with Fibrosis during Colorectal ESD
8 Imai K, Hotta K, Yamaguchi Y, Kakushima N, Tanaka M, Takizawa K, et al. Preoperative indicators of failure of en bloc resection or perforation in colorectal endoscopic submucosal dissection: implications for lesion stratification by technical difficulties during stepwise training. Gastrointest Endosc. 2016;83(5): 954-62.

9 Yoshida N, Wakabayashi N, Kanemasa K, Sumida Y, Hasegawa D, Inoue K, et al. Endoscopic submucosal dissection for colorectal tumors: technical difficulties and rate of perforation. Endoscopy. 2009;41(9):758-61.

10 Hayashi N, Tanaka S, Nishiyama S, Terasaki M, Nakadoi K, Oka S, et al. Predictors of incomplete resection and perforation associated with endoscopic submucosal dissection for colorectal tumors. Gastrointest Endosc. 2014; 79(3):427-35.

11 Sato K, Ito S, Kitagawa T, Kato M, Tominaga $\mathrm{K}$, Suzuki T, et al. Factors affecting the technical difficulty and clinical outcome of endoscopic submucosal dissection for colorectal tumors. Surg Endosc. 2014;28(10):2959-65.

12 Tanaka S, Kashida H, Saito Y, Yahagi N, Yamano H, Saito S, et al. JGES guidelines for colorectal endoscopic submucosal dissection/ endoscopic mucosal resection. Dig Endosc. 2015;27(4):417-34

13 Field MJ, Lohr KN, editors. Clinical practice guidelines: directions for a new program. Institute of Medicine (US) Committee to advise the public health service on clinical practice guidelines. Washington, DC: National Academies Press; 1990.

14 Shiga H, Endo K, Kuroha M, Kakuta Y, Takahashi S, Kinouchi Y, et al. Endoscopic submu- cosal dissection for colorectal neoplasia during the clinical learning curve. Surg Endosc. 2015;28(7):2120-8

15 Matsumoto A, Tanaka S, Oba S, Kanao H, Oka S, Yoshihara M, et al. Outcome of endoscopic submucosal dissection for colorectal tumors accompanied by fibrosis. Scand J Gastroenterol. 2010;45(11):1329-37.

16 Tanaka S, Oka S, Kaneko I, Hirata M, Mouri $\mathrm{R}$, Kanao $\mathrm{H}$, et al. Endoscopic submucosal dissection for colorectal neoplasia: possibility of standardization. Gastrointest Endosc. 2007;66(1):100-7.

17 Japanese Society for Cancer of the Colon and Rectum. Japanese classification of colorectal carcinoma. 2nd English ed. Tokyo: Kanehara \& Co., Ltd.; 2009.

18 Kim EK, Han DS, Ro Y, Eun CS, Yoo KS, Oh YH. The submucosal fibrosis: what does it mean for colorectal endoscopic submucosal dissection? Intest Res. 2016;14(4):358-64.

19 Lee SP, Kim JH, Sung IK, Lee SY, Park HS, Shim CS, et al. Effect of submucosal fibrosis on endoscopic submucosal dissection of colorectal tumors: pathologic review of 173 cases. J Gastroenterol Hepatol. 2015;30(5): 872-8.

$20 \mathrm{Fu} \mathrm{K}$, Sano Y, Kato S, Fujii T, Iwasaki J, Sugito $\mathrm{M}$, et al. Hazards of endoscopic biopsy for flat adenoma before endoscopic mucosal resection. Dig Dis Sci. 2005;50(7):1324-7.

21 Han KS, Sohn DK, Choi DH, Hong CW, Chang HJ, Lim SB, et al. Prolongation of the period between biopsy and EMR can influence the nonlifting sign in endoscopically resectable colorectal cancers. Gastrointest Endosc. 2008;67(1):97-102. 
22 Chiba H, Tachikawa J, Arimoto J, Ashikari K, Kuwabara $\mathrm{H}$, Nakaoka M, et al. Predictive factors of mild and severe fibrosis in colorectal endoscopic submucosal dissection. Dig Dis Sci. 2020;65(1):232-42.

23 Takeuchi Y, Iishi H, Tanaka S, Saito Y, Ikematsu $\mathrm{H}$, Kudo SE, et al. Factors associated with technical difficulties and adverse events of colorectal endoscopic submucosal dissection: retrospective exploratory factor analysis of a multicenter prospective cohort. Int J Colorectal Dis. 2014;29(10):1275-84.
24 Fukunaga S, Nagami Y, Shiba M, Sakai T, Maruyama H, Ominami M, et al. Impact of preoperative biopsy sampling on severe submucosal fibrosis on endoscopic submucosal dissection for colorectal laterally spreading tumors: a propensity score analysis. Gastrointest Endosc. 2019;89(3):470-8.

25 Inada Y, Yoshida N, Kugai M, Kamada K, Katada K, Uchiyama K, et al. Prediction and treatment of difficult cases in colorectal endoscopic submucosal dissection. Gastroenterol Res Pract. 2013;2013:523084.

26 Goto SP, Sakamoto N, Mitomi H, Murakami $\mathrm{T}$, Ritsuno H, Ueyama H, et al. Histological distinction between the granular and nongranular types of laterally spreading tumors of the colorectum. Gastroenterol Res Pract. 2014;2014:153935.
27 Nagai K, Hayashi Y, Honma K, Sakatani A, Yoshii S, Fujinaga T, et al. Adenoma of colorectal laterally spreading tumor nongranular type with biological phenotypic features similar to cancer. J Gastroenterol Hepatol. 2018;33(11):1853-63.

28 Kato S, Fu KI, Sano Y, Fujii T, Saito Y, Matsuda $\mathrm{T}$, et al. Magnifying colonoscopy as a non-biopsy technique for differential diagnosis of non-neoplastic and neoplastic lesions. World J Gastroenterol. 2006;12(9):1416-20.

29 Higashi R, Uraoka T, Kato J, Kuwaki K, Ishikawa S, Saito Y, et al. Diagnostic accuracy of narrow-band imaging and pit pattern analysis significantly improved for less-experienced endoscopists after an expanded training program. Gastrointest Endosc. 2010; 72(1):127-35. 